\title{
On Broca, brain, and binding: a new framework
}

\section{Peter Hagoort}

F.C. Donders Centre for Cognitive Neuroimaging \& NICl, Radboud University Nijmegen, The Netherlands

In speaking and comprehending language, word information is retrieved from memory and combined into larger units (unification). Unification operations take place in parallel at the semantic, syntactic and phonological levels of processing. This article proposes a new framework that connects psycholinguistic models to a neurobiological account of language. According to this proposal the left inferior frontal gyrus (LIFG) plays an important role in unification. Research in other domains of cognition indicates that left prefrontal cortex has the necessary neurobiological characteristics for its involvement in the unification for language. I offer here a psycholinguistic perspective on the nature of language unification and the role of LIFG.

\section{Introduction}

In this article I will outline a new general framework for a model of the neural architecture of language, with a focus on the role of Broca's area. The framework connects psycholinguistically motivated processing components to their neuronal substrate, guided by knowledge about brain function across domains of cognition. The aim is to account for neuroimaging studies on language from the design perspective rather than the experimental task perspective (e.g. phoneme identification, rhyme judgment, verb generate task, lexical decision, etc.). I believe that to make sense of the rapidly increasing number of imaging studies on language, a design perspective is urgently needed. This perspective requires a specification of the processing requirements for mapping sound onto meaning (when listening), or vice versa (when speaking).

\section{The design stance components}

From a design stance, one can distinguish three functional components of language processing: Memory, Unification and Control. The Memory component comprises a specification of the different types of language information stored in long-term memory, as well as the retrieval operations. The Unification component refers to the integration of lexically retrieved information into a representation of multi-word utterances. The Control component relates language to action, and is invoked, for instance, when the correct target language has to be selected (in the case of bilingualism), or for handling turn taking during conversation.

\footnotetext{
Corresponding author: Hagoort, P. (peter.hagoort@fcdonders.ru.nl).
}

Available online 27 July 2005
In principle, the new MUC (Memory, Unification, Control) framework applies to both language production and language comprehension, although details of their functional anatomy within each component will be different. I will first sketch the psycholinguistic motivation behind the MUC framework.

\section{Psycholinguistic background}

In psycholinguistic models of language processing, there is a general agreement that the language system has a tripartite architecture (cf. [1-3]), with levels of sound, syntax and meaning as the core aspects of our language faculty. For all these levels the following dichotomy applies: the basic information components are retrieved from long-term memory (the mental lexicon in psycholinguistic terms), whereas additional information is derived from combinatorial operations (unification) that assemble the basic components into larger structures.

Most current models of language processing agree that, in on-line sentence processing, different types of constraints are very quickly taken into consideration during speaking and listening (or reading). Constraints on how words can be structurally combined operate alongside qualitatively distinct constraints on the combination of word meanings, on the grouping of words into phonological and intonational phrases, and on their referential binding into a discourse model (see Figure 1).

Moreover, in recent linguistic theories, the distinction between lexical items and traditional rules of grammar is vanishing. For instance, Jackendoff proposes that the only remaining rule of grammar is 'UNIFY PIECES, and all the pieces are stored in a common format that permits unification.' ([2], p. 180). The unification operations clip together lexicalized patterns containing one or more variables. The operation MERGE in Chomsky's Minimalist Program [4] plays a similar role.

Although the tripartite architecture holds equally for language production and language comprehension, in the remainder of this article I will focus on language comprehension, as this has been the subject of most neuroimaging studies of language. Moreover, the focus will be on unification, because this is what Broca's area and adjacent cortex are especially relevant for.

\section{Syntactic unification}

Classically, accounts of unification in language focus on syntactic analysis. Here, again, a distinction can be made between the retrieval of syntactic frames from memory 
(a) Phonological structure

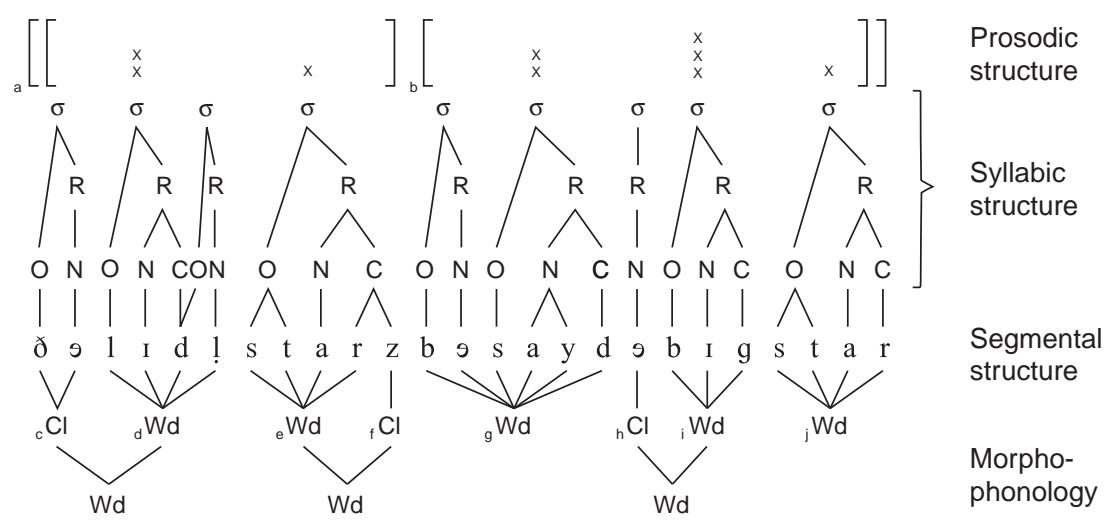

(b) Syntactic structure

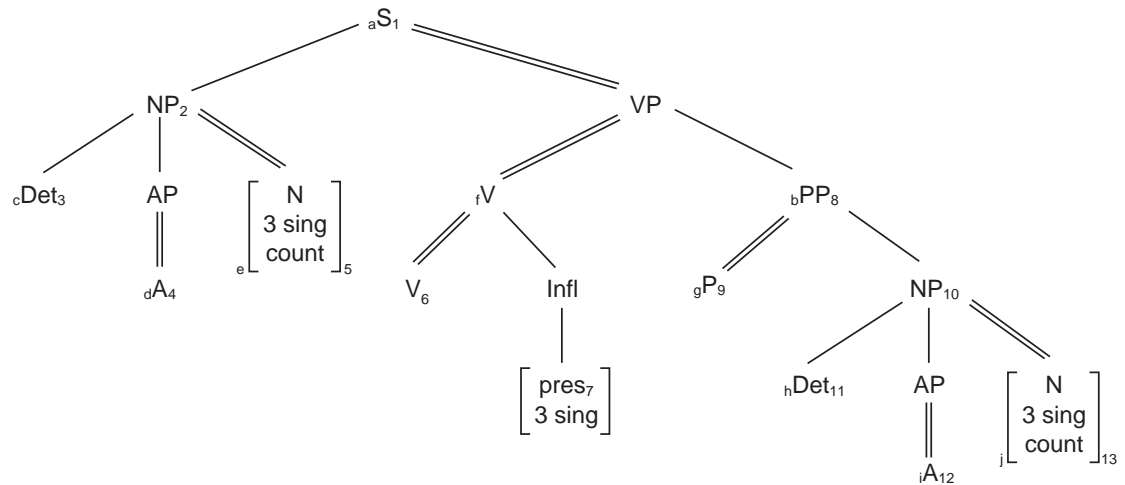

(c) Semantic/conceptual structure

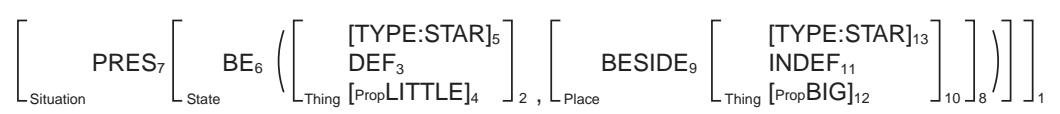

(d)

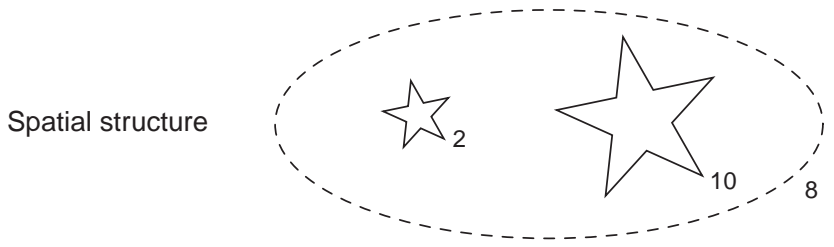

Figure 1. The tripartite architecture of the language system. The example gives the phonological (a), syntactic (b) and semantic/conceptual (c) structures for the sentence 'The little star's beside the big star'. (d) shows an approximate spatial structure of the reference objects. Reprinted from [1] with permission of Oxford University Press.

and their unification. The account of syntactic unification as proposed in the MUC framework [5] is inspired by Aravind Joshi's Tree-Adjoining Grammars and a related explicit computational model of syntactic processing [6]. In this proposal, each word form in the mental lexicon (Memory) is associated with a structural frame. This structural frame consists of a three-tiered tree, specifying the possible structural environment of the particular lexical item (see Figure 2). The top layer of the frame consists of a single phrasal node (e.g. NP). This so-called root node is connected to one or more functional nodes (e.g. Subject, Head, Direct Object) in the second layer of the frame. The third layer contains again phrasal nodes to which lexical items or other frames can be attached.

This parsing account is 'lexicalist' in the sense that all syntactic nodes (e.g. S, NP, VP, N, V, etc.) are retrieved from the mental lexicon. In other words, chunks of syntactic structure are stored in memory and there are no syntactic rules that introduce additional nodes.

In the on-line comprehension process, lexical items are retrieved sequentially, driven by the time course of the input. The structural frames associated with the individual word forms thus enter the unification workspace incrementally, in the order that is imposed by the input. In this workspace constituent structures spanning the whole 


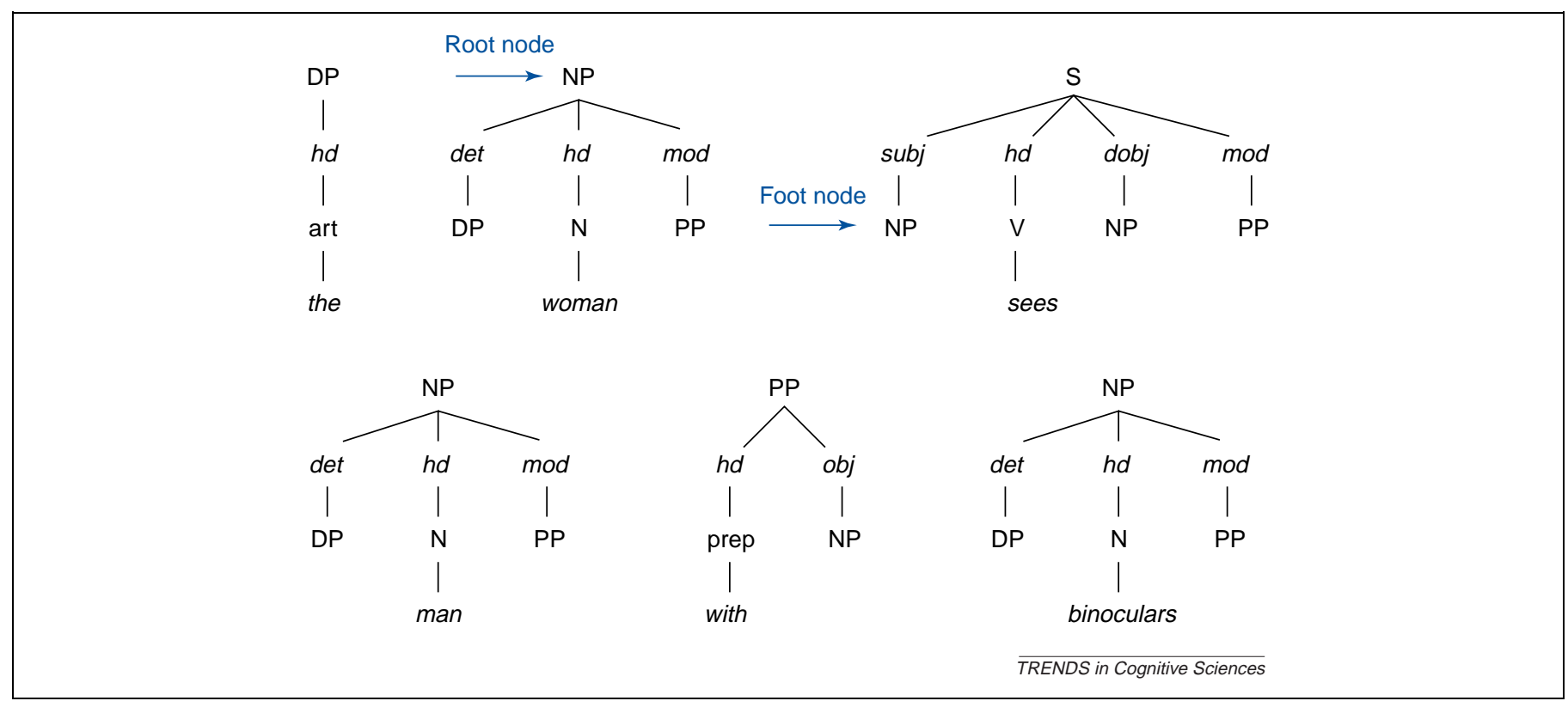

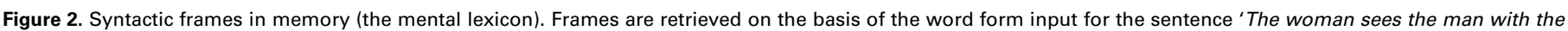

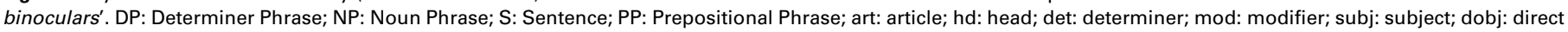

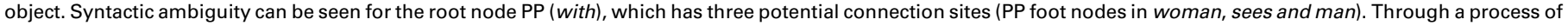
lateral inhibition, one of these PP foot nodes gets selected for unification with the PP root node.

utterance are formed by a unification operation. This operation consists of linking up lexical frames with identical root and foot nodes, and checking agreement features (number, gender, person, etc.).

The resulting unification links between lexical frames are formed dynamically, which implies that the strength of the unification links varies over time until a state of equilibrium is reached. Because of the inherent ambiguity in natural language, alternative binding candidates will usually be available at various points in the parsing process (see Figure 2). Typically, one phrasal configuration results, and this requires that among the alternative binding candidates only one remains active. This is achieved through a process of lateral inhibition between two or more alternative unification links. The outcome of the unification process is thus achieved via a selection mechanism (i.e. lateral inhibition) that 'chooses' between different unification options.

The advantage of this unification account is that: (i) it is computationally explicit, (ii) it is compatible with a large series of empirical findings in the sentence processing literature, and in the neuropsychological literature on aphasia [6], and (iii) it belongs to the class of lexicalist parsing models that have found increasing support in recent years $[2,7-9]$.

\section{Semantic and phonological unification}

Unification operations take place not only at the syntactic processing level. Combinatoriality is a hallmark of language across representational domains (cf. [2]). Thus, also at semantic and phonological levels, lexical elements are combined and integrated into larger structures. An example of semantic unification is the integration of word meaning into an unfolding discourse representation of the preceding context. For instance, the majority of common English words have more than one meaning (see www.wordsmyth.net). In the interaction with the preceding sentence or discourse context, the appropriate meaning is selected, so that a coherent interpretation results.

At the level of phonology, lexical elements are unified into intonational phrases, which are the parts of speech that are spanned by one intonational contour. The intonational phrase ends with a high or low boundary tone and is further marked by pausing, lengthening or segmental variation [10]. The characteristics of the intonational phrase co-determine which aspects of the utterance get focus. This again, cannot be determined on the basis of information retrieved from memory, but requires an analysis of how lexical elements are unified into phonological structures spanning a multi word utterance $[2,3]$.

Although explicit computational unification models are less well developed for semantics and phonology than for syntax, unification is as relevant for semantics and phonology as it is for syntax. There are good reasons to assume that in language comprehension syntactic, semantic and phonological unification processes operate concurrently and interact to some extent $[2,6]$.

\section{Neurobiological requirements for unification in language}

The need for combining independent elements into a coherent overall representation is not unique for language comprehension. It also holds for the visual system. In visual neuroscience this is referred to as the 'binding problem'. However, a major difference between object perception and language comprehension is that visual binding is more or less instantaneous (in the order of a few hundred milliseconds; [11]), whereas language comprehension is extended over time (in the order of seconds). Crucially, one core feature of the binding problem for 
language is how information that is not only processed in different parts of cortex (as in visual processing), but also at different time scales and at relatively widely spaced parts of the time axis, can be unified into a coherent representation of a multi-word utterance. A neurobiologically plausible model of language, therefore, presupposes the availability of cortical tissue that is particularly suited for maintaining information on-line while unification operations take place. As we will see, prefrontal cortex seems to be especially well-suited for doing exactly this. As I will argue below, Broca's area and adjacent cortex have the right kind of neurobiological properties to play a crucial role in unification.

\section{Broca's area extended}

Broca's area is a crucial component in all classical neurobiological models of language. However, there is no neuroanatomically motivated reason to restrict languagerelevant frontal cortex to the ill-defined region that is given the name 'Broca's area'.

Despite some disagreement in the literature (see [12]), most authors agree that Broca's area comprises Brodmann's areas (BA) 44 and 45 of the left hemisphere. At the macroscopic level these areas involve the pars opercularis (BA 44) and the pars triangularis (BA 45) of the third frontal convolution. However, detailed cytoarchitectonic analysis [13] shows that the borders of areas 44 and 45 do not neatly coincide with the sulci that were assumed to form their boundaries in macroscopic anatomical terms. More fundamentally, one has to ask what the justification is to subsume these two cytoarchitectonic areas under the overarching heading of Broca, rather than, say, Brodmann's areas 45 and 47 . Areas 44 and 45 show several clear cytoarchitectonic differences, one of which is that 45 has a granular layer IV, whereas 44 is dysgranular. In addition, areas 44 and 45 show a clear difference in their patterns of lateral asymmetry [13]. Brodmann's area 47 on the other hand is, like BA 45, part of the heteromodal component of the frontal lobe, known as the granular cortex [14].

From a neuroanatomical perspective, there thus seems to be no strong motivation to treat Broca's area as a natural kind. There is no (yet) convincing neuroanatomical evidence that requires BA 44 and BA 45 to be seen as one unified area, motivated from a cytoarchitectonic and receptor-architectonic point of view.

With respect to language areas in the left frontal lobe, it has become clear that, in addition to BA 44 and 45 , at least BA 47 and, probably, the ventral part of BA 6 should be included in the left frontal language network [15-17]. From a functional-anatomical perspective it therefore makes sense to refer to the left inferior frontal gyrus (LIFG) as the language-relevant part of frontal cortex (see Figure 3).

The argument that I will propose takes as its starting point that at a general level Broca's area might share functionality with the rest of prefrontal cortex.

\section{Prefrontal cortex function}

Integration is an important part of prefrontal cortex function. This holds especially for integration of information in the time domain $[18,19]$. To fulfill this role,

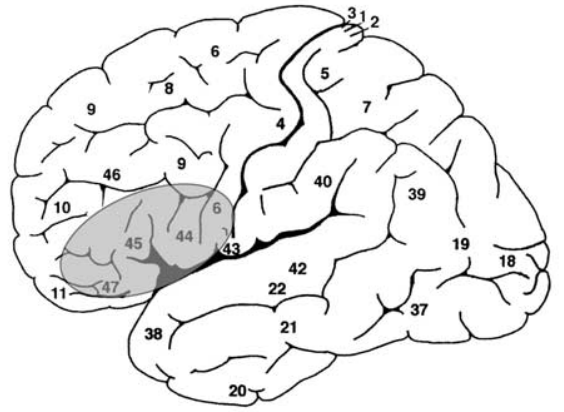

Figure 3. Lateral view of the left hemisphere. Brodmann's areas (BA) are marked by number. Classically, Broca's area comprises BA 44 and BA 45. Adjacent languagerelevant cortex also includes BA 47 and ventral BA 6 (grey oval).

prefrontal cortex needs to be able to hold information online [14], and to select among competing alternatives [20].

Electrophysiological recordings in the macaque monkey have shown that this area is important for sustaining information triggered by a transient event for many seconds [21]. This allows prefrontal cortex to select among and to establish unifications between pieces of information that are perceived or retrieved from memory at different moments in time. Recent neuroimaging studies (see below) indicate that the prefrontal cortex part of LIFG is crucial for the unification operations required for binding single word information into larger structures.

\section{LIFG as the unifcation space for language}

Results from neuroimaging studies are compatible with the proposal that the contribution of LIFG to language processing can be specified in terms of unification operations. In short, the left inferior frontal cortex recruits lexical information, mainly stored in temporal lobe structures that are known to be involved in lexical processing [22], and unifies them into overall representations that span multi-word utterances.

For syntactic unification, supporting evidence comes from numerous PET and fMRI studies (cf. [23]). In a recent meta-analysis of 28 neuroimaging studies, Indefrey [24] found two areas that were crucial for syntactic processing, independent of the input modality (visual in reading, auditory in speech). These two areas were the left posterior superior temporal gyrus and the left posterior inferior frontal cortex. The left posterior temporal cortex is known to be involved in lexical processing. This part of the brain might be important for the retrieval of the syntactic frames that are stored in the lexicon. I suggest that the unification space where individual frames are connected into a phrasal configuration for the whole utterance has the LIFG as a crucial node.

Empirical support for the role of LIFG in semantic unification has also been obtained. Hagoort et al. [17] 

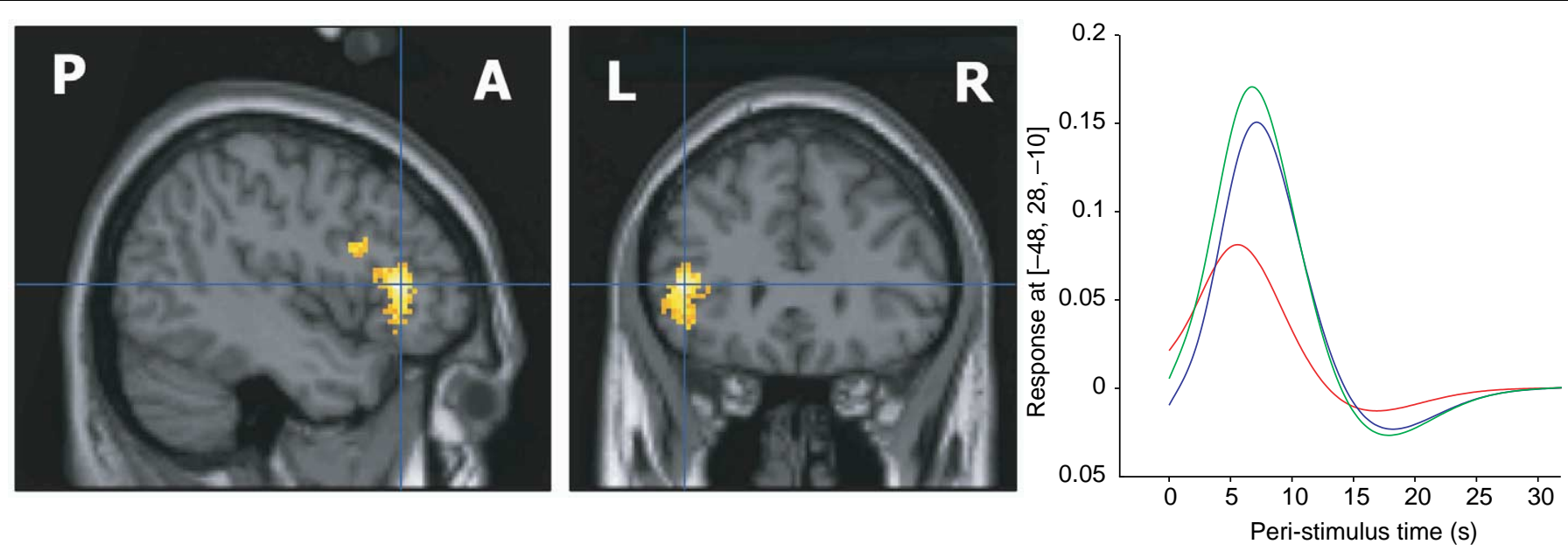

$\overline{\text { TRENDS in Cognitive Sciences }}$

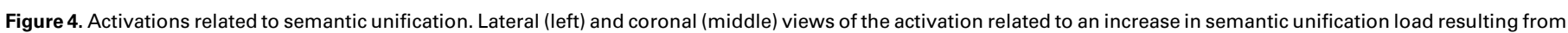

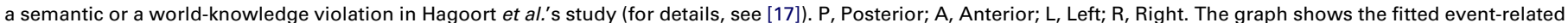

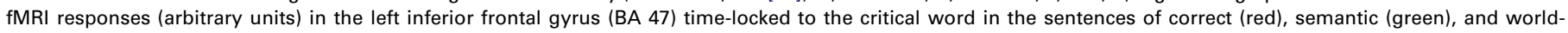

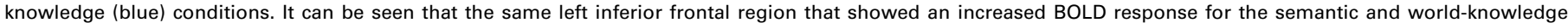

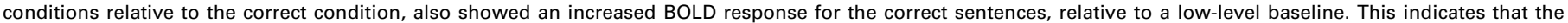
semantic and world-knowledge violations increased the unification load, but that the same area is also involved in semantic unification for correct sentences.

varied the semantic unification load in sentences containing incorrect information ('Dutch trains are white and very crowded') or semantic oddities ('Dutch trains are sour and very crowded'). In LIFG (BA 47/45; see Figure 4) an increased BOLD response was observed relative to a correct control sentence ('Dutch trains are yellow and very crowded'). This suggests that the semantic unification area is involved in establishing both the sense and the reference of an utterance. Importantly, relative to a low level baseline, increased activation was also seen for the correct sentences, indicating that this area is automatically recruited during semantic unification (see Figure 4).

Rodd et al. [25] found increased activity in BA 45 for sentences that contained ambiguous words relative to sentences with mostly unambiguous words. Sentences containing ambiguous words have a higher semantic unification load because stronger contextual selection is required to achieve a coherent interpretation.

Phonological tasks are also found to activate left frontal cortex areas, more in particular BA 44 and ventral BA 6 (cf. [15]). However, to date no studies are available that explicitly investigated phonological unification more specifically. The hypothesis is that this part of frontal cortex will be involved, but this hypothesis needs further testing.

\section{Unification gradient in LIFG}

With respect to the different unification operations within the domain of language, LIFG seems to show a functionally defined anterior-ventral to posterior-dorsal gradient (see Figure 5). Roughly speaking, BA 47 and BA 45 are involved in semantic processing; BA 45 and 44 contribute to syntactic processing; and, finally, BA 44 and parts of BA 6 have a role in phonological processing. LIFG is thus involved in at least three different domains of language processing (semantic, syntactic, phonological), with, presumably, a certain level of specialization in different LIFG subregions. However, the overlap of activations for these three different types of information is substantial and suggests the possibility of interactive concurrent processing in which various types of processing constraints are incorporated as soon as they become available.

\section{Broca's area revisited}

As I have tried to make clear, despite the historical appeal of Broca's area, this is not a very well defined concept. Instead of Broca's area, I have used the term LIFG to refer to a series of related but anatomically distinct areas, at least encompassing BA 47, 45, and 44. This set of areas

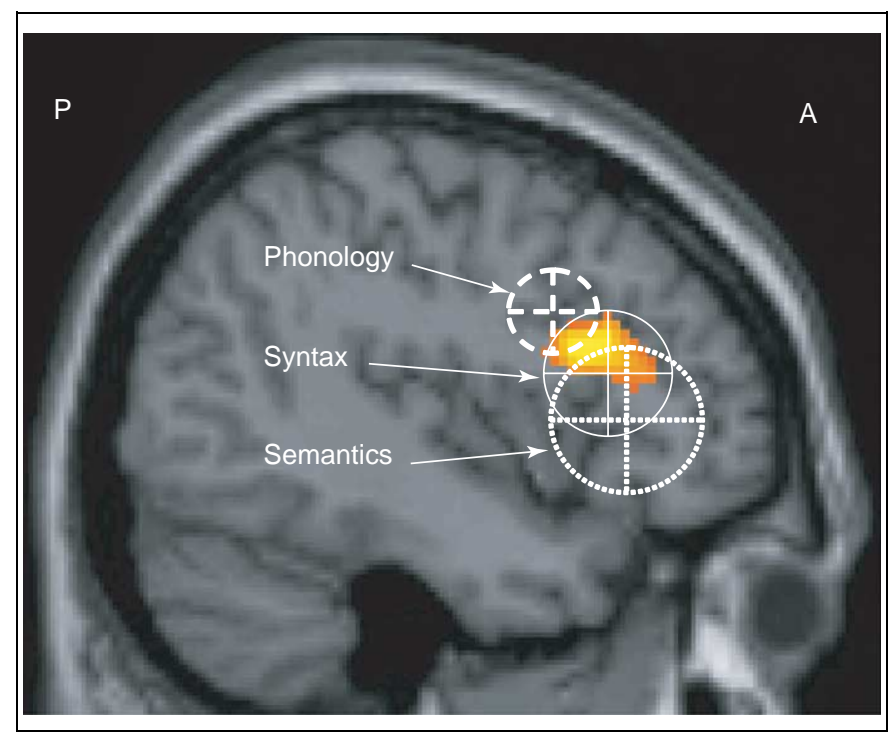

Figure 5. The unification gradient in left inferior frontal cortex. Activations and their distribution are shown, related to semantic, syntactic and phonological processing. Areas are based on the meta-analysis in Bookheimer [15]. The centres represent the mean coordinates of the local maxima, the radii represent the standard deviations of the distance between the local maxima and their means. The activation shown is from artificial grammar violations in Petersson et al. [48] (courtesy of Karl Magnus Petersson). 
subserves more than one function in the language domain, and presumably has non-language functions as well (see Box 1). Moreover, LIFG contributes to the unification of information from language with concomitant non-linguistic information, such as co-speech gestures [26].

In the context of language processing, the common denominator of Broca's area and adjacent cortex is its role in selection and unification operations by which individual pieces of lexical information are bound together into representational structures spanning multi-word utterances. One can thus conclude that Broca's area plays a pivotal role in solving one particular aspect of the binding problem for language, namely to unify lexical as well as non-linguistic information (e.g. gestures or world knowledge from long-term memory) into representations of multi-word utterances. This contribution is embedded in a network of brain areas that subserves the additional components in the design of the language system, which, according to the MUC framework, are memory and control. I will now briefly discuss the neural basis of the other two components.

\section{The Memory and Control components}

The left temporal cortex plays a crucial role in storage and retrieval of linguistic information that language acquisition has laid down in memory. This refers to the phonological/phonetic properties of words, their syntactic features such as grammatical gender, word class (verb, noun, etc.) including the syntactic frames, and finally the conceptual specifications of the 60000 or so words that a native speaker of a language such as English has stored in memory. Activations related to phonological/phonetic properties are reported for the central to posterior superior temporal gyrus (STG) extending into the superior temporal sulcus (STS) $[22,27,28]$.

Semantic information is presumably distributed over several brain areas, but most likely different parts of the left middle and inferior temporal gyri are crucial for lexical-semantic processing [22,29-33].

\section{Box 1. Specificity of Broca's area}

Although Broca's area and adjacent cortex play an important role in unification within the domain of language, this doesn't mean that it is a language-specific area, or that within the language domain it subserves only one function. As Mesulam has argued [41,42], 'many cortical nodes are likely to participate in the function of more than one network.' ([41], p.1040). In this conception of brain mechanisms, a particular cognitive function is served by a distributed network of areas, rather than by one local area alone. In addition, the local area participates in more than one function. For instance, Broca's area has also been found to be activated when subjects had to search for a target hidden within a complex geometric pattern [43], during action recognition [44,45], and during movement preparation [46]. Of course, all this does not mean that cognitive functions are not localized at all, or that the brain shows equipotentiality. It only means that the one-area, one-function principle is in many cases not an adequate account of how cognitive functions are neuronally instantiated. Even for the visual system it is claimed that the representations of, for example, objects and faces in ventral temporal cortex are distributed and overlapping [47]. It would be highly surprising if the language network in the brain behaved according to more localist principles than the visual system.
Hardly anything is known about the brain areas involved in the lexical retrieval of a word's syntactic specifications. On the basis of the meta-analysis of a large series of imaging studies on syntactic processing [24], the hypothesis is that the left posterior superior temporal cortex (Wernicke's area) is involved in the retrieval of lexical-syntactic information. The Memory component thus seems to be distributed mainly over the (left) temporal cortex.

The Control component accounts for the fact that the language system operates in the context of communicative intentions and actions. For example, attentional control allows individuals to speak while seeing relevant and irrelevant objects or hearing interlocutors, to take turns in conversational settings, or in case of bilingualism to select the correct language in a particular communicative setting. The issue of verbal control has so far only been studied in the context of a Stroop task [34-37]. These studies suggest that a network of areas consisting of the anterior cingulate cortex (ACC) and the dorsolateral prefrontal cortex (DLPC, BA 46/9) is involved in verbal action planning and attentional control.

Figure 6 summarizes the network of areas subserving the three central components (Memory, Unification and Control) of human language in action. The precise effective connectivity between these areas is a topic for further research.

\section{The MUC framework in relation to other proposals}

A small number of neurobiologically inspired models of the human language faculty have been proposed in recent years $[30,38,39]$. Some of these models are more restricted than the MUC framework and focus mainly on one aspect of language processing, such as single word processing [30,40], or grammar and morphology [39]. An alternative broad scope model [38] attributes a mainly strategic role to LIFG. By contrast, according to the MUC framework, strategic effects are especially related to the Control

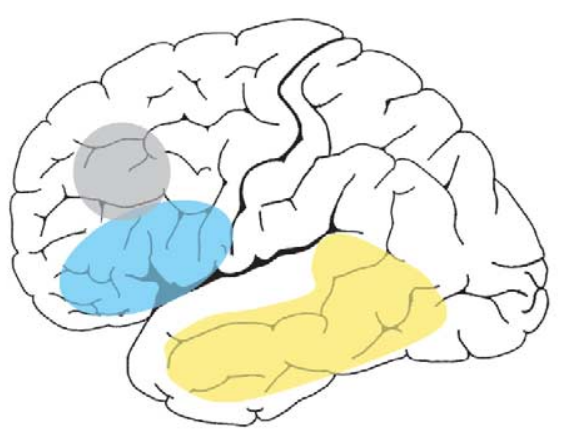

Figure 6. The three components of the MUC model projected onto a lateral surface of the left hemisphere: Memory (yellow) in left temporal cortex, Unification (blue) in LIFG, and Control (grey) in dorsolateral prefrontal cortex. The anterior cingulate cortex (ACC; part of the Control component) is not shown. 


\section{Box 2. Questions for future research}

- What is the role of the right hemisphere, and subcortical structures such as thalamus, basal ganglia and cerebellum, in memory, unification and control operations for language?

- How are the interfaces between semantic, syntactic and phonological unification operations organized in computational and neuronal terms?

- What are the precise computational characteristics of semantic and phonological unification?

- Which of the neural structures in LIFG and left temporal cortex are shared by language production and language comprehension?

- Is unification restricted to LIFG, or do other areas participate to some extent?

component, but the role of LIFG in language processing is mandatory. In addition, the MUC model assumes partly interactive and concurrent unification for phonology, syntax and semantics, without an informational/temporal precedence for syntax.

\section{Conclusions}

Most accounts of the contribution of Broca's area to language start from a task perspective (phoneme identification, verb generation, etc.). Here I have taken a design stance that distinguishes three core components for language processing: memory, unification and control. I have argued that the neurobiological requirements for unification are those of working memory, which include that lexical building blocks are kept activated for some time while unification operations take place. Broca's area and adjacent cortex seem to meet these requirements. Within LIFG, semantic, syntactic and phonological unification operations can be partly dissociated. The characterization of phonological and semantic unification in relation to LIFG function especially needs further investigation (see Box 2).

\section{Acknowledgements}

I thank Peter Indefrey, Willem Levelt, Karl Magnus Petersson, Aslı Özyürek, Ivan Toni, Roel Willems and the anonymous referees for their helpful comments on an earlier version of the manuscript.

\section{References}

1 Jackendoff, R. (1999) The representational structures of the language faculty and their interactions. In The Neurocognition of Language (Brown, C.M. and Hagoort, P., eds), pp. 37-79, Oxford University Press

2 Jackendoff, R. (2002) Foundations of Language: Brain, Meaning, Grammar, Evolution, Oxford University Press

3 Levelt, W.J.M. (1999) Producing spoken language: A blueprint of the speaker. In The Neurocognition of Language (Brown, C.M. and Hagoort, P., eds), pp. 83-122, Oxford University Press

4 Chomsky, N. (1995) The Minimalist Program, MIT Press

5 Hagoort, P. (2003) How the brain solves the binding problem for language: a neurocomputational model of syntactic processing. Neuroimage 20, S18-S29

6 Vosse, T. and Kempen, G.A.M. (2000) Syntactic structure assembly in human parsing: a computational model based on competitive inhibition and lexicalist grammar. Cognition 75, 105-143

7 Bresnan, J.W. (2001) Lexical-Functional Syntax, Blackwell

8 Joshi, A.K. and Schabes, Y. (1997) Treeadjoining grammars. In Handbook of Formal Languages and Automata (Vol. 3) (Salomma, A. and Rosenberg, G., eds), pp. 69-124, Springer-Verlag

9 MacDonald, M.C. et al. (1994) Lexical nature of syntactic ambiguity resolution. Psychol. Rev. 101, 676-703
10 Cutler, A. and Clifton, C.E. (1999) Comprehending spoken language: A blueprint of the listener. In The Neurocognition of Language (Brown, C.M. and Hagoort, P., eds), pp. 123-166, Oxford University Press

11 Varela, F. et al. (2001) The brainweb: Phase synchronization and largescale integration. Nat. Rev. Neurosci. 2, 229-239

12 Uylings, H.B.M. et al. (1999) Broca's language area from a neuroanatomical and developmentsl perspective. In The Neurocognition of Language (Brown, C.M. and Hagoort, P., eds), pp. 319-336, Oxford University Press

13 Amunts, K. et al. (1999) Broca's area revisited: cytoarchitecture and intersubject variability. J. Comp. Neurol. 412, 319-341

14 Mesulam, M-M. (2002) The human frontal lobes: Transcending the default mode through contingent encoding. In Principles of Frontal Lobe Function (Stuss, D.T. and Knight, R.T., eds), pp. 8-31, Oxford University Press

15 Bookheimer, S. (2002) Functional MRI of Language: New approaches to understanding the cortical organization of semantic processing. Annu. Rev. Neurosci. 25, 151-188

16 Devlin, J.T. et al. (2003) Semantic processing in the left prefrontal cortex: A combined functional magnetic resonance imaging and transcranial magnetic stimulation study. J. Cogn. Neurosci. 15, 71-84

17 Hagoort, P. et al. (2004) Integration of word meaning and world knowledge in language comprehension. Science 304, 438-441

18 Fuster, J.M. (1995) Temporal processing. Ann. N. Y. Acad. Sci. 769, 173-181

19 Fuster, J.M. (2002) Physiology of executive functions: The perceptionaction cycle. In Principles of Frontal Lobe Function (Stuss, D.T. and Knight, R.T., eds), pp. 96-108, Oxford University Press

20 Thompson-Schill, S.L. et al. (1999) Effects of repetition and competition on activity in left prefrontal cortex during word generation. Neuron 23, 513-522

21 Miller, E.K. (2000) The prefrontal cortex and cognitive control. Nat. Rev. Neurosci. 1, 59-65

22 Indefrey, P. and Cutler, A. (2005) Prelexical and lexical processing in listening. In The Cognitive Neurosciences (Gazzaniga, M.S., ed.), pp. 759-774, MIT Press

23 Kaan, E. and Swaab, T. (2002) The brain circuitry of syntactic comprehension. Trends Cogn. Sci. 6, 350-356

24 Indefrey, P. (2004) Hirnaktivierungen bei syntaktischer Sprachverarbeitung: eine Meta-Analyse. In Neurokognition der Sprache (Mueller, H.M. and Rickheit, S., eds), pp. 31-50, Stauffenburg Verlag

25 Rodd, J.M. et al. The neural mechanisms of speech comprehension: fMRI studies of semantic ambiguity. Cereb. Cortex (in press)

26 Willems, R. et al. (2005) The comprehension of gesture and speech. J. Cogn. Neurosci. (Suppl.) 17, 231

27 Scott, S.K. and Johnsrude, I.S. (2003) The neuroanatomical and functional organization of speech perception. Trends Neurosci. 26, 100-107

28 Aleman, A. et al. (2005) The functional neuroanatomy of metrical stress evaluation of perceived and imagined spoken words. Cereb. Cortex 15, 221-228

29 Damasio, H. et al. (1996) A neural basis for lexical retrieval. Nature 380, 499-505

30 Hickok, G. and Poeppel, D. (2000) Towards a functional neuroanatomy of speech perception. Trends Cogn. Sci. 4, 131-137

31 Indefrey, P. and Levelt, W.J.M. (2000) The neural correlates of language production. In The New Cognitive Neurosciences (Gazzaniga, M.S., ed.), pp. 845-865. MIT Press

32 Indefrey, P. and Levelt, W.J.M. (2004) The spatial and temporal signatures of word production components. Cognition 92, 101-144

33 Saffran, E. and Sholl, A. (1999) Clues to the functional and neural architecture of word meaning. In The Neurocognition of Language (Brown, C.M. and Hagoort, P., eds), pp. 241-273, Oxford University Press

34 Botvinick, M.M. et al. (2004) Conflict monitoring and anterior cingulate cortex: an update. Trends Cogn. Sci. 8, 539-546

35 Bush, G. et al. (2000) Cognitive and emotional influences in anterior cingulate cortex. Trends Cogn. Sci. 4, 215-222

36 MacLeod, C.M. and MacDonald, P.A. (2000) Interdimensional interference in the Stroop effect: Uncovering the cognitive and neural anatomy of attention. Trends Cogn. Sci. 4, 383-391

37 Roelofs, A. and Hagoort, P. (2002) Control of language use: cognitive modeling the hemodynamics of Stroop task performance. Brain Res. Cogn. Brain Res. 15, 85-97 
38 Friederici, A.D. (2002) Towards a neural basis of auditory sentence processing. Trends Cogn. Sci. 6, 78-84

39 Ullman, M.T. (2001) A neurocognitive perspective on language: the declarative/procedural model. Nat. Rev. Neurosci. 2, 717-726

40 Hickok, G. and Poeppel, D. (2004) Dorsal and ventral streams: a framework for understanding aspects of the functional anatomy of language. Cognition 92, 67-99

41 Mesulam, M-M. (1998) From sensation to cognition. Brain 121, 1013-1052

42 Mesulam, M-M. (1990) Large-scale neurocognitive networks and distributed processing for attention, language, and memory. Ann. Neurol. 28, 597-613

43 Fink, G.R. et al. (in press) A role for Broca's area beyond language processing: evidence from neuropsychology and fMRI. In Broca's area (Amunts, K. and Grodzinsky, Y., eds), Oxford University Press

44 Decety, J. et al. (1997) Brain activity during observation of actions. Influence of action content and subject's strategy. Brain 120, 1763-1777

45 Hamzei, F. et al. (2003) The human action recognition system and its relationship to Broca's area: An fMRI study. Neuroimage 19, 637-644

46 Thoenissen, D. et al. (2002) Differential involvement of parietal and precentral regions in movement preparation and motor intention. J. Neurosci. 22, 9024-9034

47 Haxby, J.V. et al. (2001) Distributed and overlapping representations of faces and objects in ventral temporal cortex. Science 293, 2425-2430

48 Petersson, K.M. et al. (2004) Artificial syntactic violations activate Broca's area. Cogn. Sci. 28, 383-407

\section{Elsevier celebrates two anniversaries with gift to university libraries in the developing world}

In 1580, the Elzevir family began their printing and bookselling business in the Netherlands, publishing works by scholars such as John Locke, Galileo Galilei and Hugo Grotius. On 4 March 1880, Jacobus George Robbers founded the modern Elsevier company intending, just like the original Elzevir family, to reproduce fine editions of literary classics for the edification of others who shared his passion, other 'Elzevirians'. Robbers co-opted the Elzevir family's old printer's mark, visually stamping the new Elsevier products with a classic old symbol of the symbiotic relationship between publisher and scholar. Elsevier has since become a leader in the dissemination of scientific, technical and medical (STM) information, building a reputation for excellence in publishing, new product innovation and commitment to its STM communities.

In celebration of the House of Elzevir's 425th anniversary and the 125th anniversary of the modern Elsevier company, Elsevier will donate books to 10 university libraries in the developing world. Entitled 'A Book in Your Name', each of the 6700 Elsevier employees worldwide has been invited to select one of the chosen libraries to receive a book donated by Elsevier. The core gift collection contains the company's most important and widely used STM publications including Gray's Anatomy, Dorland's IIlustrated Medical Dictionary, Essential Medical Physiology, Cecil Essentials of Medicine, Mosby's Medical, Nursing and Allied Health Dictionary, The Vaccine Book, Fundamentals of Neuroscience, and Myles Textbook for Midwives.

The 10 beneficiary libraries are located in Africa, South America and Asia. They include the Library of the Sciences of the University of Sierra Leone; the library of the Muhimbili University College of Health Sciences of the University of Dar es Salaam, Tanzania; the library of the College of Medicine of the University of Malawi; and the libraries of the University of Zambia, Universite du Mali, Universidade Eduardo Mondlane, Mozambique; Makerere University, Uganda; Universidad San Francisco de Quito, Ecuador; Universidad Francisco Marroquin, Guatemala; and the National Centre for Scientific and Technological Information (NACESTI), Vietnam.

Through 'A Book in Your Name', the 10 libraries will receive approximately 700 books at a retail value of approximately 1 million US dollars.

$$
\text { For more information, visit www.elsevier.com }
$$

